\title{
Spontaneous remission of a large thyroid tumour
}

\author{
Kenta Watanabe MD PhD, Koichi Kajiwara MD
}

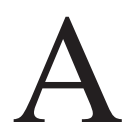

67-year-old woman with a history of rheumatoid arthritis treated with sulfasalazine and methotrexate for two and a half years presented with a one-month history of neck swelling. Her cumulative methotrexate dose was about $800 \mathrm{mg}$. Computed tomography (CT) showed a large mass involving the left lobe of her thyroid, along with lymphadenopathy (Figure 1A and B) and esophageal compression (Figure 1B). An incisional thyroid biopsy suggested diffuse large B-cell lymphoma. Although serology results were consistent with prior infection with Epstein-Barr virus, results from in situ hybridization of thyroid tissue were negative for the virus. We diagnosed methotrexate-related lymphoma. After withdrawal of methotrexate, the swelling of the patient's neck gradually abated. No other treatments were used. Follow-up CT at one and a half months showed a dramatic resolution of lesions (Figure 1C and D). Complete remission was reconfirmed at a two-year follow-up visit, at which time our patient was taking only sulfasalazine for her rheumatoid arthritis.

The overall standardized incidence ratio for lymphoma in patients with rheumatoid arthritis has been estimated at 1.9 (95\% confidence interval 1.3-2.7), which is similar to the incidence ratio for patients receiving methotrexate monotherapy. ${ }^{1}$ Cases of methotrexate-associated lymphoma resolving with discontinuation of the drug have been described, both in the presence and absence of latent infection with Epstein-Barr virus. ${ }^{2-4}$ Our case represents a possible adverse reaction to methotrexate based on the Naranjo scale for probability of an adverse drug reaction. ${ }^{5}$ Physicians prescribing methotrexate for rheumatoid arthritis may wish to keep in mind its associations with lymphoproliferative disorder.

\section{References}

1. Wolfe F, Michaud K. Lymphoma in rheumatoid arthritis: the effect of methotrexate and anti-tumor necrosis factor therapy in 18,572 patients. Arthritis Rheum 2004;50:1740-51.
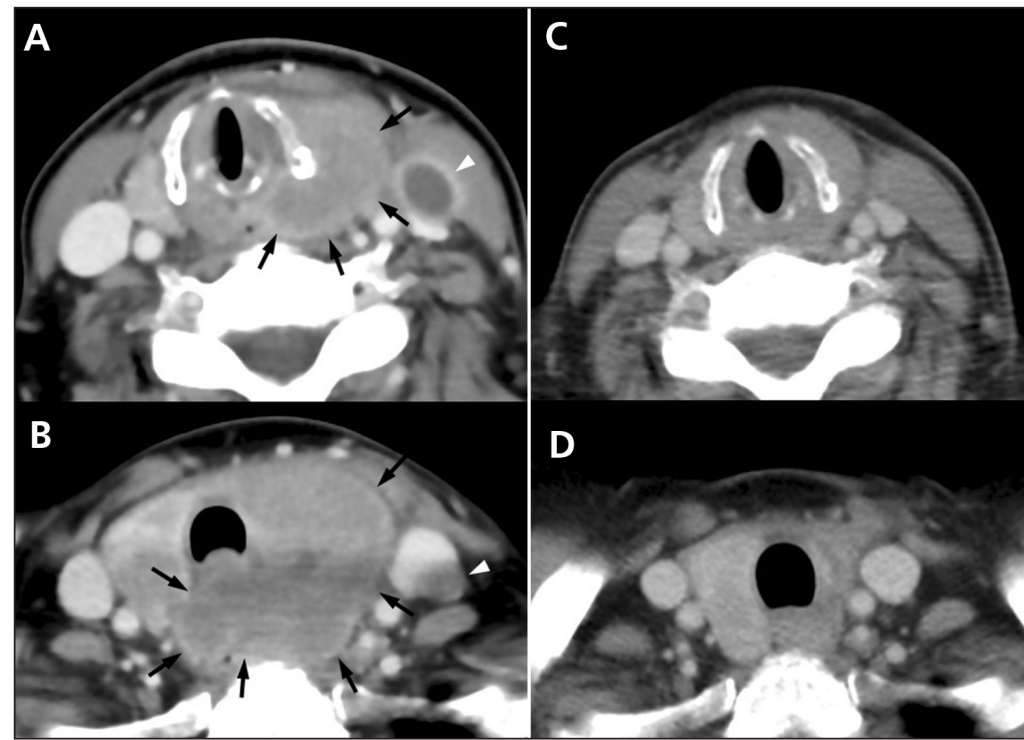

D

Figure 1: Computed tomographic (CT) images at (A) the level of the cricoid cartilage and (B) the level of the cervical trachea of the neck of a 67-year-old woman, showing a large mass involving the left lobe of the thyroid gland (arrows) and the cricoid cartilage. Lymphadenopathy (white arrowheads) and mass effect on the esophagus are also evident. (C and D) Follow-up CT images showing resolution of the thyroid mass and lymphadenopathy one and a half months following withdrawal of methotrexate.

2. Mariette X, Cazals-Hatem D, Warszawki J, et al. Lymphomas in rheumatoid arthritis patients treated with methotrexate: a 3-year prospective study in France. Blood 2002;99:3909-15.

3. Miyazaki T, Fujimaki K, Shirasugi Y, et al. Remission of lymphoma after withdrawal of methotrexate in rheumatoid arthritis: relationship with type of latent Epstein-Barr virus infection. Am J Hematol 2007;82:1106-9.

4. Hoshida Y, Xu JX, Fujita S, et al. Lymphoproliferative disorders in rheumatoid arthritis: clinicopathological analysis of 76 cases in relation to methotrexate medication. J Rheumatol 2007;34:322-31.

5. Naranjo CA, Busto U, Sellers EM, et al. A method for estimating the probability of adverse drug reactions. Clin Pharmacol Ther 1981;30:239-45.

Clinical images are chosen because they are particularly intriguing, classic or dramatic. Submissions of clear, appropriately labelled high-resolution images must be accompanied by a figure caption and the patient's written consent for publication. A brief explanation (250 words maximum) of the educational significance of the images with minimal references is required.
Competing interests: None declared.

This article has been peer reviewed.

The authors have obtained patient consent.

Affiliations: Department of Otolaryngology (Watanabe), Toranomon Hospital; Department of Hematology (Kajiwara), Tokyo Metropolitan Tama Medical Center, Tokyo, Japan

Correspondence to:

Kenta Watanabe,

Quentaw@aol.com

CMAJ 2016. DOI:10.1503 /cmaj.150400 